\title{
Field-angle-dependent specific heat measurements and gap determination of a heavy fermion superconductor $\mathrm{URu}_{2} \mathrm{Si}_{2}$
}

\author{
K. Yano, ${ }^{1}$ T. Sakakibara, ${ }^{1}$ T. Tayama,${ }^{1}$ M. Yokoyama,${ }^{2}$ H. Amitsuka,${ }^{3}$ \\ Y. Homma, ${ }^{4}$ P. Miranović, ${ }^{5}$ M. Ichioka, ${ }^{6}$ Y. Tsutsumi,${ }^{6}$ and K. Machida ${ }^{6}$ \\ 1 Institute for Solid State Physics, University of Tokyo, Kashiwa, Chiba 270-8581, Japan \\ 2 Faculty of Science, Ibaraki University, Mito 310-8512, Japan \\ 3 Graduate School of Science, Hokkaido University, Sapporo 060-0810, Japan \\ 4 IMR Tohoku University, Oarai 311-1313, Japan \\ 5 Department of Physics, University of Montenegro, Podgorica 81000, Montenegro \\ 6 Department of Physics, Okayama University, Okayama 700-8530, Japan
}

(Dated: October 29, 2018)

\begin{abstract}
To identify the superconducting gap structure in $\mathrm{URu}_{2} \mathrm{Si}_{2}$ we perform field-angle-dependent specific heat measurements for the two principal orientations in addition to field rotations, and theoretical analysis based on microscopic calculations. The Sommerfeld coefficient $\gamma(H)$ 's in the mixed state exhibit distinctively different field-dependence. This comes from point nodes and substantial Pauli paramagnetic effect of $\mathrm{URu}_{2} \mathrm{Si}_{2}$. These two features combined give rise to a consistent picture of superconducting properties, including a possible first order transition of $H_{\mathrm{c} 2}$ at low temperatures.
\end{abstract}

PACS numbers: $74.70 . \mathrm{Tx}, 74.25 . \mathrm{Bt}, 74.25 . \mathrm{Op}$

It is believed that heavy fermion superconductors (SCs), because of their heavy effective mass arising from strong electron correlation effects, mostly exhibit unconventional pairing states other than a $s$-wave pairing to avoid strong on-site repulsion. Among various known materials $\mathrm{URu}_{2} \mathrm{Si}_{2}$ is relatively old, discovered in 1985 [1], and has yet full of mysteries. A phase transition at $T_{\mathrm{o}}=17.5 \mathrm{~K}$ which was thought to be an antiferromagnetic order with a tiny moment [2], is now under lively debate on its origin [3]. Under this so-called "hidden order" (HO) the superconducting state appears at $T_{\mathrm{c}}=1.3 \mathrm{~K}$.

The specific heat $C(T) \propto T^{2}$ at low $T[\underline{4},[\underline{5}$ ] and nuclear relaxation rate $T_{1}^{-1} \propto T^{3}$ [6] suggest a line node gap or more accurately that the density of states (DOS) at low energy $N(E) \propto|E|$. Up to now, further details of the pairing symmetry in $\mathrm{URu}_{2} \mathrm{Si}_{2}$ remain unknown: Where the line node is if any, or other alternatives.

Usually by a bulk thermodynamic measurement alone, it is impossible to locate the position of nodes on the Fermi surface (FS), except for using the field-angledependent methods $7,8,8,10,11$, 12]. For example, $d_{x y}$ and $d_{x^{2}-y^{2}}$-wave gap structures can be distinguished by rotating an external field $H$ relative to crystal axis. The maximum (minimum) of the oscillation amplitude in the Sommerfeld coefficient $\gamma(H)$ in low- $T$ specific heat [8, 9, 11] or thermal conductivity $\kappa(H) / T$ [12] corresponds to the anti-nodal (nodal) direction [13]. Note that $\lim _{T \rightarrow 0} C(H) / T=\gamma(H)=\frac{2}{3} \pi^{2} \hbar^{2} N(0, H)$, where $N(0, H)$ is the zero energy DOS (ZEDOS) at the Fermi level.

In this Letter, using the field-angle-dependent specific heat measurement, we try to clarify the gap structure of superconductivity in $\mathrm{URu}_{2} \mathrm{Si}_{2}$. For the identification of the point nodes located at north and south poles on the FS in tetragonal crystal, simple measurement by the field rotation is not enough, because the twofold oscil- lation pattern due to large tetragonal anisotropy of the upper critical field $H_{\mathrm{c} 2}$ hinders the gap structure. Therefore, as an extension of the angle-dependent methods, we examine the $H$ dependence of $\gamma(H)$ for each field orientation, as shown in Fig. 1. To identify the point nodes, we use a specialty of them [8]. As seen in Fig. 1)(b), $\gamma(H)$ behaves differently on $H \| c$ and $H \| a$, because main contributions to $\gamma(H)$ come from the excited quasiparticles (QPs) with the Fermi momentum perpendicular to $H$. Namely, $\gamma_{c}(H) \propto H$ for $H \| c$ where the existing point nodes are not sensed by the excited QPs, yielding a slow rise in $\gamma_{c}(H)$. This linear $\gamma_{c}(H)$ resembles that expected for the full gap structure [14]. On the other hand, $\gamma_{a}(H)$ for $H \| a$ shows a steep increase at lower $H$, because the point nodes are effectively sensed by the excited QPs for this $H$ direction. This $\gamma_{a}(H)$ behavior is similar to the
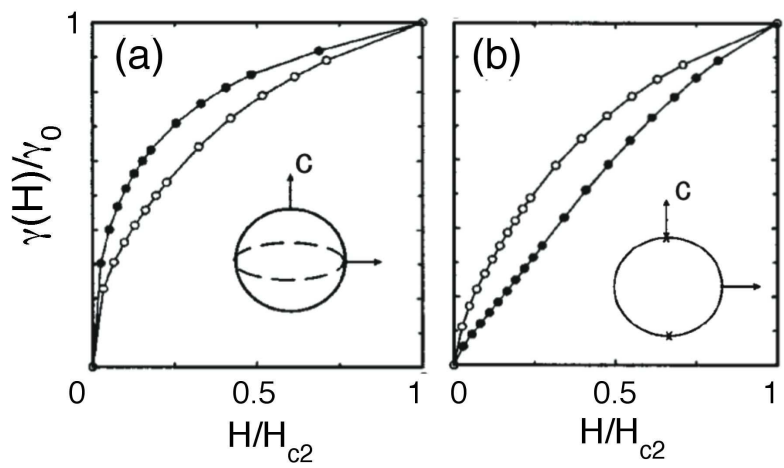

FIG. 1: $H$-dependence of low- $T \gamma(H)$ for $H \| c(\bullet)$ and $H \| a(\circ)$, calculated by quasiclassical theory [8]. We plot $\gamma(H) / \gamma_{0}$ vs $H / H_{c 2}$ for the line node on the equator (a) and point nodes on the poles (b), as schematically shown there. $\gamma_{0}$ denotes the Sommerfeld coefficient in the normal state. 


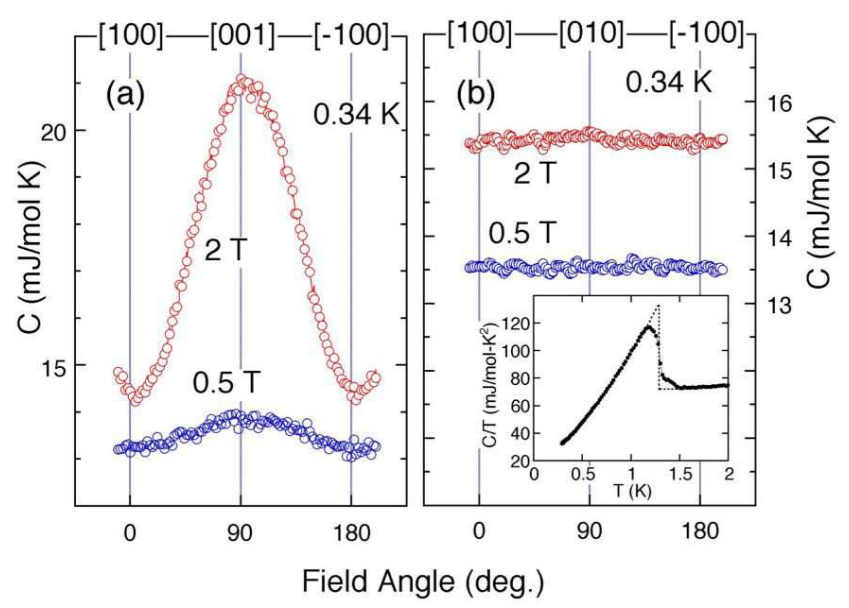

FIG. 2: (Color online) $C(H, \theta)$ of $\mathrm{URu}_{2} \mathrm{Si}_{2}$ at $T=0.34 \mathrm{~K}$. The field-rotational plane is perpendicular to [010] (a) and to [001] (b) directions. Inset shows $C / T$ vs. $T$ plot at $H=0$.

so-called Volovik $\sqrt{H}$ dependence [15]. This feature is absent in the line node case in Fig. 1(a), because the excited QPs feel more or less the nodal structure for both $H$ orientations, leading to essentially the same behavior $\gamma_{a, c}(H) \propto \sqrt{H}$ with slightly different coefficient.

Through our analysis, it will also become clear that the Pauli paramagnetic depairing effect is operative for both directions in $\mathrm{URu}_{2} \mathrm{Si}_{2}$. Previously two experiments point out this fact; a strong depression of $H_{c 2}$ [16] and decreasing tendency of the Maki parameter $\kappa_{2}$ upon cooling for both directions [17, 18]. The following experiment and analyses reinforce this, suggesting a possible first order phase transition at lower $T[19]$.

Experiment. We have carried out the angle-dependent specific heat measurements $C(H, \theta)$ on a single crystal $\mathrm{URu}_{2} \mathrm{Si}_{2}$ (100 mg weight), grown by the Czochralski method and annealed in high vacuum for 7 days at $950^{\circ} \mathrm{C}$. The crystal had $T_{\mathrm{c}}$ of $1.3 \mathrm{~K}$ as shown in the inset of Fig. 2. $C(H, \theta)$ measurements were carried out by means of a semi-adiabatic method in horizontal fields up to $7 \mathrm{~T}$ at a lower $T$ of $0.34 \mathrm{~K}[11$.

In Fig. 2 the data $C(H, \theta)$ are shown under the field rotated in the (010) (Fig. 2(a)) and (001) plane (Fig. 2(b)). The former exhibits a large twofold oscillation, which is mainly due to a large anisotropy in $H_{\mathrm{c} 2}\left(\mu_{0} H_{\mathrm{c} 2}^{a} \simeq\right.$ $13 \mathrm{~T}, \mu_{0} H_{\mathrm{c} 2}^{c} \simeq 3 \mathrm{~T}$ for $\left.T \rightarrow 0\right)$. It should be noticed that minima of $C(H, \theta)$ in Fig. 2(a) do not correspond to nodal directions. Unfortunately, any subtle oscillation of $C(H, \theta)$ in the $a c$-plane that could result from nodal structures is washed out by the strong anisotropy in $H_{\mathrm{c} 2}$. Thus, we have to examine the $H$-dependence of $C(H, \theta)$ at each field orientation.

We have also searched for a possible line nodal structure running parallel to the $c$ axis, which can be detected by rotating $H$ within the (001) plane. As shown in Fig. 2(b) there is no apparent oscillation within the experi-
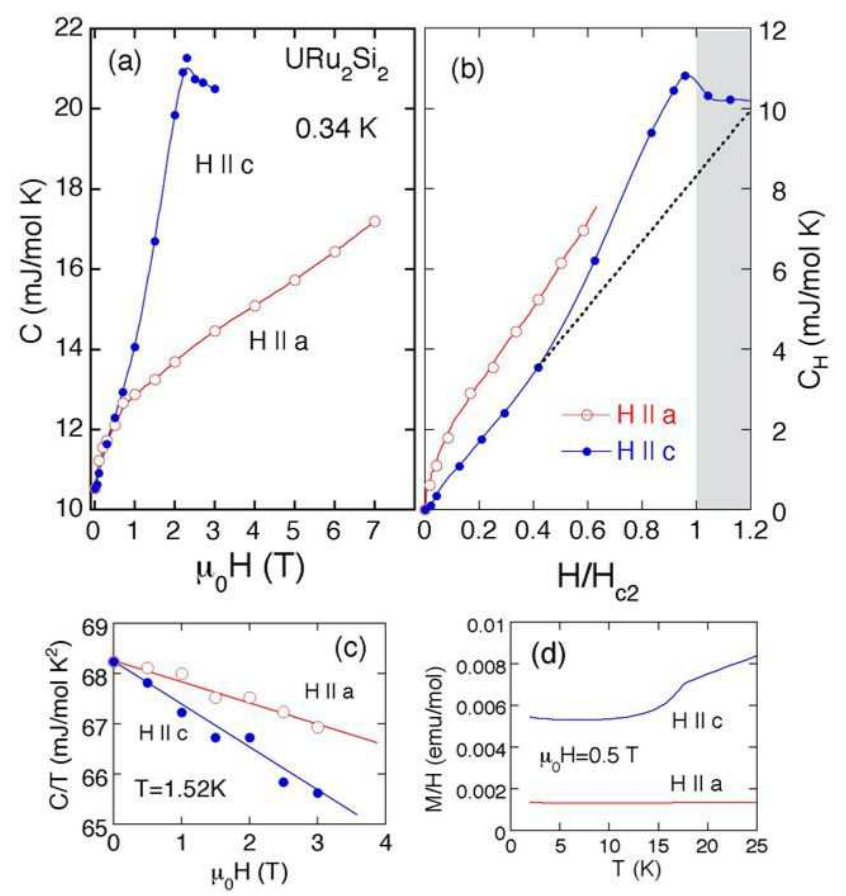

FIG. 3: (Color online) (a) Field variation of $C(H)$ of $\mathrm{URu}_{2} \mathrm{Si}_{2}$ at $T=0.34 \mathrm{~K}$ for $H \| a$ (open circles) and $H \| c$ (solid circles). Solid lines are guides to the eyes. (b) $H$-dependent part of $C$ as a function of $H / H_{\mathrm{c} 2}$. The dotted line is a linear extrapolation of the low-field part for $H \| c$. (c) $H$-variation of $C$ in the normal state at $1.52 \mathrm{~K}$ for $H \| a$ and $H \| c$. (d) $T$-variation of $M / H$ in the normal state below $25 \mathrm{~K}$ for $H \| a$ and $H \| c$.

mental accuracy. We could conclude that there is no line node parallel to the $c$ axis because the expected oscillation amplitude $(3 \sim 4 \%)[8,9]$ is beyond the experimental resolution $(\sim 0.3 \%)$. However this cannot exclude the possibility for the line node along the equator on the FS and weaker gap anisotropy parallel to the $c$ axis.

In order to investigate the possible nodal structure, we examined the field variation of the specific heat in detail. Figure 3(a) displays the results at $T=0.34 \mathrm{~K}$ for both $H \| a$ and $H \| c . C_{\mathrm{c}}(H)$ for $H \| c$ peaks at $H_{\mathrm{c} 2}^{c}(\sim 2.5 \mathrm{~T})$ and reaches the normal-state value at higher $H$. For $H \| a$, in contrast, $C_{\mathrm{a}}(H)$ continues to increase at $7 \mathrm{~T}$ because $H_{\mathrm{c} 2}^{a}(\sim 12.5 \mathrm{~T})$ is much higher in this direction. Accordingly, a significant anisotropy in $C_{\mathrm{a}, \mathrm{c}}(H)$ develops at high $H$. Surprisingly, this anisotropy in $C_{\mathrm{a}, \mathrm{c}}(H)$ rapidly diminishes with decreasing $H$ and both curves almost fall on top of each other below $0.5 \mathrm{~T}$. This unexpected behavior can be seen more clearly in Fig. 3(b), where the field dependent part of $C$ is plotted as a function of $H / H_{\mathrm{c} 2}$ for the two directions. It is remarkable to find the distinctively different field dependence of $C_{\mathrm{a}, \mathrm{c}}\left(H / H_{\mathrm{c} 2}\right)$ at low $H$. The rapid rise in $C_{\mathrm{a}}\left(H / H_{\mathrm{c} 2}\right)$ is reminiscent of a nodal structure. $C_{\mathrm{c}}\left(H / H_{\mathrm{c} 2}\right)$ shows, in contrast, a much weaker linear rise, suggesting a full gap feature. This distinctive $C(H)$ behavior for two directions never oc- 
curs when the line nodes exist on the FS, as discussed in Fig. 11(a). Thus, the experimental characteristics of $\mathrm{URu}_{2} \mathrm{Si}_{2}$ in Fig. 3(b) are qualitatively consistent with the gap structure with the point nodes locating at the north and south poles on the FS, as shown in Fig. 1(b).

Since superconductivity in $\mathrm{URu}_{2} \mathrm{Si}_{2}$ coexists with $\mathrm{HO}$ that sets in at $17.5 \mathrm{~K}$, one might suspect that its field-direction dependent excitations could result in the anisotropic behavior of $C_{\mathrm{a}, \mathrm{c}}\left(H / H_{\mathrm{c} 2}\right)$ at low $H$. In order to rule out this possibility, we carried out the $C(H)$ measurements at $1.52 \mathrm{~K}$ just outside the superconducting state but still well inside the $\mathrm{HO}$ phase (Fig. 3) (c)). For both field directions, $C_{\mathrm{a}, \mathrm{c}}(H) / T$ is only weakly $H$ dependent; a slight linear decrease by $2 \sim 3 \%$ has been observed at $2 \mathrm{~T}$. Since the $C_{\mathrm{a}, \mathrm{c}}(H) / T$ variation in the superconducting state (Fig. 3)(a)) is much larger $(40 \sim 100 \%$ at $2 \mathrm{~T}$ ), this change in the normal-state background is negligible. In order to back up this argument, we have also measured the magnetization $M(T)$ at $\mu_{0} H=0.5 \mathrm{~T}$ (Fig. 3(d)). It is well known that $M_{\mathrm{a}}(T)$ for $H \| a$ is nearly $T$-independent whereas $M_{\mathrm{c}}(T)$ for $H \| c$ exhibits a kink upon $\mathrm{HO}$ at $17.5 \mathrm{~K}$ and rapidly decreases at lower $T$ [1]. For both directions, $M / H$ below $10 \mathrm{~K}$, where HO is well developed, is virtually $T$-independent. The slight upturn in $M_{\mathrm{a}, \mathrm{c}}(T)$ seen below $5 \mathrm{~K}$ in Fig. 3(d) is presumably due to impurity effect. Then making use of a Maxwell relation $\partial^{2} M / \partial T^{2}=T^{-1} \partial C / \partial H$, field variation of $C(H)$ is expected to be small, consistent with the results in Fig. 3(c). Thus we conclude that the contribution of $\mathrm{HO}$ to $\mathrm{C}(H)$ is small and negligible; the distinctively different field dependence of $\mathrm{C}_{\mathrm{a}, \mathrm{c}}\left(\mathrm{H} / \mathrm{H}_{\mathrm{c} 2}\right)$ in Fig. 3(b) arises from superconductivity in the system.

Compared with theoretical curves in Fig. 1(b), $\gamma(H)$ of the experimental data in Fig. 3(b) is suppressed at the middle- $H$ region for both directions. Especially for $H \| c$, starting from a gradual linear increase (dotted line) at low $H, \gamma(H)$ shows concave curvature at high $H$, which is in contrast to the theoretical curve in Fig. 1(b) showing convex curvature. We may attribute the anomalous behavior to the Pauli paramagnetic depairing that suppresses superconductivity eminently at high $H$. Then $H_{\mathrm{c} 2}$ is reduced, and $\gamma(H)$ rapidly rises at high $H$.

According to the $M(H)$ measurements [17, 18], the Maki parameter $\kappa_{2} \propto 1 / \sqrt{(\partial M / \partial H)}_{H=H_{c 2}}$ is an increasing function of $T$ for both orientations, which is opposite to an ordinary $\mathrm{SC}[20]$. At $H=H_{\mathrm{c} 2}$ they show large paramagnetic moments build in the mixed state. These facts clearly indicate that the paramagnetic effect is an important ingredient in fully understanding this material.

Theoretical calculation. To support above suggestions, we calculate the ZEDOS $N(0, H)$ by the microscopic quasi-classical theory in the clean limit including the paramagnetic effect. As is done in Ref. [21], we selfconsistently determine the spatial structure of the pair potential $\Delta(\mathbf{r})$ and the vector potential $\mathbf{A}(\mathbf{r})$, to appropriately evaluate the vortex core contribution, through the

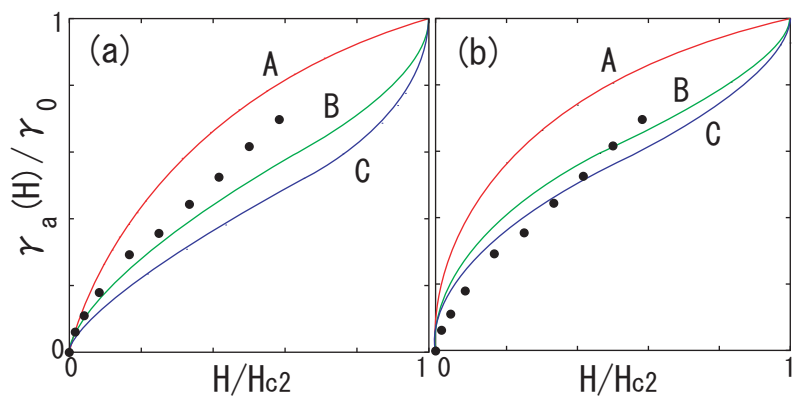

FIG. 4: (Color online) $H$-dependence of $\gamma(H)$ for $H \| a$ in the linear point node case $\phi(\mathbf{k})=\sin \theta($ a) and the quadratic point node case $\phi(\mathbf{k})=\sin ^{2} \theta(\mathrm{b})$. Solid circles are the experimental data in Fig. 3(b). $\mu=0$ (A), 0.85 (B), 1.7 (C) in (a). $\mu=0$ (A), $0.6(\mathrm{~B}), 1.0(\mathrm{C})$ in (b)

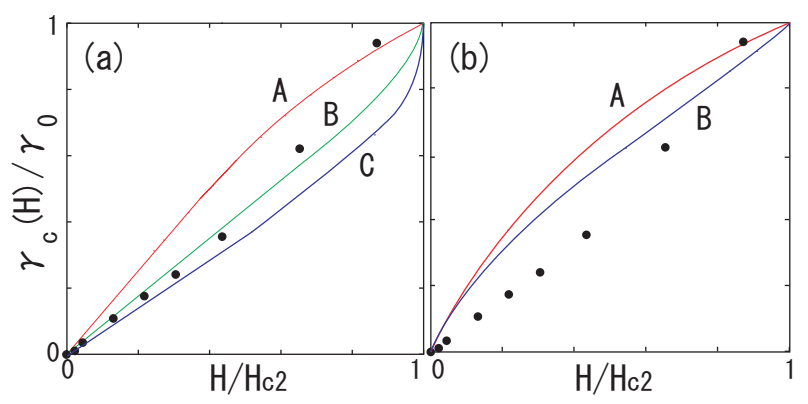

FIG. 5: (Color online) The same as in Fig. 4 but for $H \| c$. $\mu=0(\mathrm{~A}), 1.6(\mathrm{~B}), 2.5(\mathrm{C})$ in (a). $\mu=0(\mathrm{~A}), 1.3(\mathrm{~B})$ in (b).

quasi-classical Green's functions $g\left(\omega_{n}, \mathbf{k}, \mathbf{r}\right), f\left(\omega_{n}, \mathbf{k}, \mathbf{r}\right)$, and $f^{\dagger}\left(\omega_{n}, \mathbf{k}, \mathbf{r}\right)$, which are calculated by the Eilenberger equation

$$
\begin{aligned}
& \left\{\omega_{n}+\mathrm{i} \mu B+\mathbf{k} \cdot(\nabla+\mathrm{i} \mathbf{A})\right\} f=\Delta \phi(\mathbf{k}) g \\
& \left\{\omega_{n}+\mathrm{i} \mu B-\mathbf{k} \cdot(\nabla-\mathrm{i} \mathbf{A})\right\} f^{\dagger}=\Delta^{*} \phi^{*}(\mathbf{k}) g
\end{aligned}
$$

with $g=\left(1-f f^{\dagger}\right)^{1 / 2}$, Reg $>0$, Matsubara frequency $\omega_{n}=(2 n+1) \pi T$, and effective Zeeman energy $\mu B$. $\mu$ characterizes the strength of the paramagnetic effect. The self-consistent calculation is performed at $T=0.1 T_{c}$ in the triangular vortex lattice for $\mathbf{k}$ on an isotropic Fermi sphere. The local DOS at an energy $E$ is given by $N(E, \mathbf{r})=\left\langle\operatorname{Re} g\left(\mathrm{i} \omega_{n} \rightarrow E+\mathrm{i} 0^{+}, \mathbf{k}, \mathbf{r}\right)\right\rangle_{\mathbf{k}}$ with the Fermi sphere average $\langle\cdots\rangle_{\mathbf{k}}$. By the spatial average of $N(E=$ $0, \mathbf{r})$, we obtain the ZEDOS $N(0, H)=\langle N(E=0, \mathbf{r})\rangle_{\mathbf{r}}$.

In Figs. 4 and 5 we examine two cases of point nodes, (i) quadratic point nodes by the pairing function $\phi(\mathbf{k})=$ $\sin ^{2} \theta$, and (ii) linear point nodes by $\phi(\mathbf{k})=\sin \theta$, where $\theta$ is the polar angle from the $c$ axis. The former (i) is consistent with the experiments $C(T) \propto T^{2}$ [4, 5] and $T_{1}^{-1} \propto T^{3}[6]$. However, as shown in Fig. [5(b), the resulting $\gamma_{c}(H)$ exhibits upward curvature at low $H$, which fails to explain the linear $H$-dependence of our experimental data. The latter (ii), used in the calculation for Fig. 1, can reproduce $\sqrt{H}$-like behavior for $H \| a$ (Fig. 4(a)) and $H$-linear behavior for $H \| c$ (Fig. 5)(a)). For 
better fitting to the experimental data, we have to include the paramagnetic effect by $\mu$. The best fittings at low $H$ are attained by $\mu_{a}=0.85\left(\mu_{c}=1.60\right)$ for $H \| a(H \| c)$. The discrepancy at higher $H$ is due to thermal effect because the experiment was not done at sufficiently low $T$. Near $T_{\mathrm{c}}(H), C(H) / T$ is larger than $\gamma(H)$. According to the $M(H)$ measurements [17, 18], the paramagnetic moments at $H_{\mathrm{c} 2}$ are comparable for two directions, i.e. $\mu_{a}^{2} H_{\mathrm{c} 2}^{a} \simeq \mu_{c}^{2} H_{\mathrm{c} 2}^{c}$. This indicates $\mu_{c} / \mu_{a}=2.1$, which is consistent to our choice $\mu_{c} / \mu_{a}=1.60 / 0.85 \sim 1.9$.

Our calculation can show that above a critical value $\mu^{*} \sim 0.4$ the first order transition occurs at $H_{c 2}$ in lower $T$ and higher $H$ for a Fermi sphere and $s$ wave pairing. Thus, our assigned $\mu$ values are within the first order region. Since the $C(H)$ data were taken at $T=0.34 \mathrm{~K}$, there is no indication for it. However, recent thermal conductivity measurements [19] clearly shows a jump of $\kappa(H)$ at $H_{\mathrm{c} 2}$ for both directions around $T \sim 0.1 \mathrm{~K}$. This is in accord with our assignment for the $\mu$ values.

Among the possible pairing function in the group theoretical classifications for a tetragonal crystal [22, 23], spin-triplet symmetries such as $\tau_{x} k_{x}+\tau_{y} k_{y}, \tau_{x} k_{x}+i \tau_{y} k_{y}$, $\tau_{z}\left(k_{x}+i k_{y}\right)$, and $\left(\tau_{x}+i \tau_{y}\right)\left(k_{x}+i k_{y}\right)$ have point nodes $\left(\tau_{j}=i \sigma_{2} \sigma_{j}\right)$, but they are unlikely to be realized in $\mathrm{URu}_{2} \mathrm{Si}_{2}$ because the paramagnetic effect is present in both directions. In spin-singlet symmetries, $k_{x}^{2}+k_{y}^{2}\left(\mathrm{~A}_{1 g}\right)$ has two quadratic point nodes, and $k_{z}\left(k_{x}+i k_{y}\right)\left(\mathrm{E}_{g}\right)$ consists of two linear point nodes and a line node. Our analysis supports linear point nodes, and excludes line nodes. Thus, if we choose $k_{z}\left(k_{x}+i k_{y}\right)$ as a plausible gap function with linear point nodes, the line node contribution may be smeared out due to the actual FS topology [24]. While we have to consider realistic FS topology for conclusive evaluation of the gap structure, the linear point nodes in the polar direction are plausible, because low- $H$ behavior of $\gamma(H)$ is governed by the low- $E$ QPs excited around nodes [15].

$\mathrm{CeCoIn}_{5}$ is also considered to be a Pauli limited SC with a tetragonal structure. The phase diagrams in $H$ vs $T$ for both field directions are similar and exhibit Paulilimited behavior. It is understandable because $M$ values at $H_{\mathrm{c} 2}$ are almost same for both $H \| c$ and $H \| a$ [25], which matters the paramagnetic effect. This situation is the same as in $\mathrm{URu}_{2} \mathrm{Si}_{2}$ as mentioned before. These two compounds belong to a clean limit SC free from dirt effects, such as spin-orbit scattering that tends to mask a first order transition due to the paramagnetic effect.

In summary, we performed field-angle-dependent specific heat measurements in $\mathrm{URu}_{2} \mathrm{Si}_{2}$. The Sommerfeld coefficient $\gamma(H)$ exhibits distinctively different behavior for $H \| c$ and $H \| a$. These reveal, supported by microscopic calculation, that the gap structure posses point nodes at north and south poles on the Fermi surface and moreover the Pauli paramagnetic effect is important in this system. This also suggests a first order transition at $H_{\mathrm{c} 2}$ in lower temperatures, which has been confirmed recently [19].

We thank Y. Matsuda for informative discussions.

[1] T.T.M. Palstra et al., Phys. Rev. Lett. 55, 2727 (1985); M.B. Maple et al., Phys. Rev. Lett. 56, 185 (1986); W. Schlabitz et al., Z. Phys. B 62, 171 (1986).

[2] C. Broholm et al., Phys. Rev. Lett. 58, 1467 (1987).

[3] C.R. Wiebe et al., Nature Phys. 3, 96 (2007); H. Amitsuka et al., J. Mag. Mag. Mater. 310, 214 (2007); Y.S. Oh et al., Phys. Rev. Lett. 98, 016401 (2007).

[4] R.A. Fisher et al., Physica B 163, 419 (1990).

[5] J.P. Brison et al., Physica B 199-200, 70 (1994).

[6] K. Matsuda et al., J. Phys. Soc. Jpn. 65, 679 (1996).

[7] I. Vekhter et al., Phys. Rev. B 59, R9023 (1999).

[8] P. Miranović et al., Phys. Rev. B 68, 052501 (2003).

[9] P. Miranović et al., J. Phys.: Condens. Matter 17, 7971 (2005).

[10] H. Adachi et al., Phys. Rev. Lett. 94, 067007 (2005).

[11] T. Sakakibara et al., J. Phys. Soc. Jpn. 76, 051004 (2007), and references therein.

[12] Y. Matsuda et al., J. Phys. Condens. Matter 18 R705 (2006), and references therein.

[13] The interpretation of the oscillation pattern in $\kappa(H) / T$ is complicated by the additional factor due to the directional dependent scattering mechanism in some case.

[14] N. Nakai et al., Phys. Rev. B 70, 100503(R) (2004).

[15] G.E. Volovik, Pis'ma Zh. Eksp. Teor. Fiz. 58, 457 (1993) [JETP Lett. 58, 469 (1993)].

[16] J.P. Brison, et al, Physica C 250, 128(1995).

[17] K. Tenya et al., Physica B 281-282, 991 (2000).

[18] K. Tenya et al., preprint.

[19] Y. Kasahara et al., Phys. Rev. Lett. 99, 116402 (2007).

[20] D. Saint-James, G. Sarma, and E.J. Thomas, Type II Superconductivity (Pergamon, Oxford, 1969), Chap. 6.

[21] M. Ichioka and K. Machida, Phys. Rev. B 76, 064502 (2007).

[22] G.E. Volovik and L.P. Gor'kov, Zh. Eksp. Teor. Fiz. 88, 1412 (1985) [Sov. Phys. JETP 61, 843 (1985)].

[23] M. Ozaki et al., Prog. Theor. Phys. 75, 442 (1986).

[24] During the preparation of the manuscript, we have learned of a paper [19], which concludes the same gap structure $k_{z}\left(k_{x}+i k_{y}\right)$ by measuring thermal conductivity. They assign the point (line) nodes on the main (minor) FS with heavy (light) mass. These are completely consistent with and reinforce our conclusions.

[25] T. Tayama et al., Phys. Rev. B 65, 180504(R) (2002). 